\title{
Trans-Modulation in Wireless Relay Networks
}

\author{
Karim G. Seddik, Ahmed S. Ibrahim, and K. J. Ray Liu
}

\begin{abstract}
In this letter, we consider the trans-modulation design for the decode-and-forward relay networks. We propose to reassign the constellation points at the relay nodes to minimize the symbol error rate (SER) at the destination node. The proposed trans-modulation scheme can significantly improve the system SER performance without increasing the complexity of the system, especially when the relays are close to the source. For this case, improvements of about $2 \mathrm{~dB}$ for 16-QAM constellation and about $3 \mathrm{~dB}$ for 64-QAM constellation are achieved for the single-relay case.
\end{abstract}

Index Terms - Constellation design, decode-and-forward protocol, wireless relay networks.

\section{INTRODUCTION}

$\mathbf{R}$ ECENTLY, the use of cooperative diversity techniques has gained a lot of interest [1], [2]. In cooperative diversity, the cooperation of several single-antenna nodes is allowed to emulate a multiple antenna transmitter. Several protocols have been proposed to achieve the gains promised by the use of node cooperation. In [1], several protocols were proposed for the single-relay case to achieve cooperative diversity; outage analyses were provided for these protocols. Among these protocols is the decode-and-forward (DAF) protocol. In the DAF protocol, the relay node decodes the received source signal before retransmission to the destination node. If the relay node always forwards the source signal then, there will be error propagation and consequently a diversity of order one is achieved, limited by errors at the relay node.

In order to achieve a diversity of order two for the singlerelay DAF protocol, the relay should be able to decide whether or not it has decoded correctly. This can be achieved through the use of error detecting codes or the use of appropriate SNR threshold at the relay node [3]. The works in [1], [3] consider the use of repetition coding, where the relay nodes just repeat the source symbols. In this letter, we consider the design of trans-modulation, e.g., the re-mapping of the constellation points at the relay nodes to minimize the destination SER. Our proposed constellation reassignment scheme can be considered as some form of complex field coding by trying to increase the Euclidean distance between the different transmitted symbols.

Constellation reassignment was considered in [4] for multiple packets transmissions over additive white Gaussian noise (AWGN) channels. The destination uses automatic repeat request (ARQ) to request retransmission of packets that were decoded erroneously. The idea is to rearrange the constellation points for the retransmitted data packets. The aim is to

Manuscript received October 20, 2007. The associate editor coordinating the review of this letter and approving it for publication was S. Buzzi.

The authors are with the Department of Electrical and Computer Engineering, and Institute for Systems Research, University of Maryland, College Park, MD 20742, USA (e-mail: \{kseddik, asalah, kjrliu\}@umd.edu).

Digital Object Identifier 10.1109/LCOMM.2008.071734.

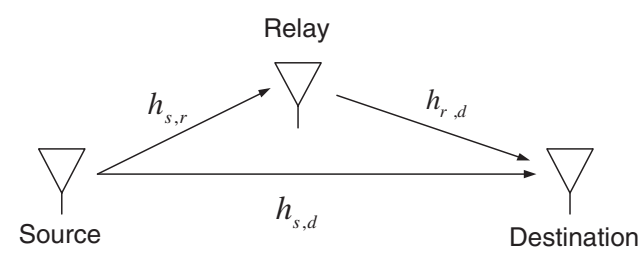

Fig. 1. Simplified system model for the single-relay channel.

minimize the destination bit error rate (BER). In [5], the idea of constellation rearrangement was implemented by using different constellation arrangements for the transmissions over different diversity branches. The authors considered the case of 16-QAM constellation and showed that constellation rearrangement can significantly improve the destination block error rate performance.

In this letter, the design of trans-modulation at the relay node to minimize the destination SER is proposed. We consider the cases of 16-QAM and 64-QAM constellations. The proposed scheme can be easily extended to higher order constellations.

\section{Single-Relay DAF Protocol System Model}

In this section, the system model for the DAF protocol is presented. For simplicity of presentation, the single-relay DAF protocol is considered, but the scheme can be easily extended to the multi-node DAF protocol. A simplified block diagram of the system is depicted in Fig. 1. The transmission protocol has two phases as follows. In phase 1, the source broadcasts its information $x_{s}$ to the destination and relay nodes. The source symbol is assumed to be carved from M-QAM or MPSK constellations. The source symbol is normalized such that $E\left\{\left|x_{s}\right|^{2}\right\}=1$, where $E\{\cdot\}$ denotes the expectation operator. The received signals $y_{s, d}$ and $y_{s, r}$ at the destination and relay nodes are given, respectively, by

$$
\begin{aligned}
& y_{s, d}=\sqrt{P_{s}} h_{s, d} x_{s}+\eta_{s, d} \\
& y_{s, r}=\sqrt{P_{s}} h_{s, r} x_{s}+\eta_{s, r},
\end{aligned}
$$

where $P_{s}$ is the transmitted source power, $\eta_{s, d}$ and $\eta_{s, r}$ denote the additive white Gaussian noise at the destination and the relay nodes, respectively, and $h_{s, d}$ and $h_{s, r}$ are the channel coefficients from the source to the destination node and the relay node, respectively.

If the relay node was able to decode correctly, it will help the source in phase 2; otherwise, it will remain idle [3]. The received signal at the destination in phase 2 , due to the relay node transmission, is given by

$$
y_{r, d}=\sqrt{P_{r}} h_{r, d} x_{r}+\eta_{r, d},
$$


where $P_{r}$ is the transmitted relay power, $\eta_{r, d}$ denotes the additive white Gaussian noise at the destination, and $h_{r, d}$ is the channel coefficient from the relay node to the destination. Here $x_{r}$ denotes the new constellation point transmitted from the relay node and is normalized such that $E\left\{\left|x_{r}\right|^{2}\right\}=1$. For the repetition based approach [1], [3], [6], the symbol $x_{r}$ is the same as $x_{s}$. The channel coefficients $h_{s, d}, h_{s, r}$, and $h_{r, d}$ are modeled as zero-mean complex Gaussian random variables with variances $\delta_{s, d}^{2}, \delta_{s, r}^{2}$, and $\delta_{r, d}^{2}$, respectively, i.e. a Rayleigh flat fading channel model is considered. The channel coefficients are assumed to be only available at the receiving nodes. The noise terms are modeled as zero-mean complex Gaussian random variables with variance $N_{0} / 2$ per dimension.

\section{TRAns-Modulation Design}

In this section, an expression for the pairwise symbol error probability (PSEP) between two possible transmitted source symbols is derived. This analysis gives a guideline on how to design the constellation points at the relay node(s) to improve the system SER performance. The PSEP at the destination node is defined as

$$
\begin{aligned}
\operatorname{Pr} & \left\{\mathbf{x}_{1} \rightarrow \mathbf{x}_{2}\right\} \\
= & \operatorname{Pr}\left\{\mathbf{x}_{1} \rightarrow \mathbf{x}_{2} \mid \mathbf{x}_{1}, \text { relay decodes erroneously }\right\} \\
& \times \operatorname{Pr}\{\text { relay decodes erroneously }\} \\
+ & \operatorname{Pr}\left\{\mathbf{x}_{1} \rightarrow \mathbf{x}_{2} \mid \mathbf{x}_{1}, \text { relay decodes correctly }\right\} \\
& \times \operatorname{Pr}\{\text { relay decodes correctly }\},
\end{aligned}
$$

where $\mathbf{x}_{1}$ and $\mathbf{x}_{2}$ are two possible transmitted source symbols. The vector $\mathbf{x}_{1}=\left[\begin{array}{ll}\sqrt{P_{s}} x_{s_{1}} & \sqrt{P_{r}} x_{r_{1}}\end{array}\right]^{T}$, where $x_{s_{1}}$ is the source transmitted constellation point and $x_{r_{1}}$ is the relay transmitted constellation point, similarly, $\mathbf{x}_{2}=\left[\begin{array}{lll}\sqrt{P_{s}} x_{s_{2}} & \sqrt{P_{r}} x_{r_{2}}\end{array}\right]^{T}$.

The PSEP expression in (4) has two terms depending on the state of the relay node (whether or not it has decoded correctly). The first term corresponds to the case when the relay decodes erroneously. This term only depends on the constellation used at the source node while the second term clearly depends on the constellations used at the source and relay nodes. We consider the design of the constellation points at the relay node in order to minimize that term.

The use of maximum likelihood (ML) detector at the receiver is assumed. We consider minimizing the term $P S E P_{r}=\operatorname{Pr}\left\{\mathbf{x}_{1} \rightarrow \mathbf{x}_{2} \mid \mathbf{x}_{1}\right.$, relay decodes correctly $\}$, which corresponds to the case when the relay correctly decodes the source symbol. Under our system model assumptions, the $P S E P_{r}$ of the ML detector can be expressed as

$$
P S E P_{r}=E\left\{\operatorname{Pr}\left\{q<0 \mid \mathbf{x}_{1} \text {, relay decodes correctly }\right\}\right\},
$$

where

$$
q=\left[\begin{array}{ll}
\mathbf{z}_{1}^{\mathcal{H}} & \mathbf{z}_{2}^{\mathcal{H}}
\end{array}\right]\left[\begin{array}{cc}
\mathbf{I}_{2} & \mathbf{0} \\
\mathbf{0} & -\mathbf{I}_{2}
\end{array}\right]\left[\begin{array}{l}
\mathbf{z}_{1} \\
\mathbf{z}_{2}
\end{array}\right]
$$

in which $\mathbf{z}_{1}=\left(\operatorname{diag}\left(\mathbf{x}_{1}\right)-\operatorname{diag}\left(\mathbf{x}_{2}\right)\right) \mathbf{h}+\mathbf{n}, \mathbf{z}_{2}=\mathbf{n}, \mathbf{h}=$ $\left[h_{s, d} h_{r, d}\right]^{T}, \mathbf{n}=\left[\begin{array}{ll}\eta_{s, d} & \eta_{r, d}\end{array}\right]^{T}$, and $\mathbf{I}_{2}$ is the $2 \times 2$ identity matrix.

It can be proved that the conditional probability density function of $q$ in (5), given the channel coefficients and given that $\mathbf{x}_{1}$ was transmitted and relay decoded correctly, is Gaussian. The $P S E P_{r}$ can be proved to be given by

$$
\begin{aligned}
& P S E P_{r}=E\{ \\
& \left.Q\left(\sqrt{\frac{1}{2 N_{0}}\left(P_{s}\left|h_{s, d}\right|^{2}\left|x_{s_{1}}-x_{s_{2}}\right|^{2}+P_{r}\left|h_{r, d}\right|^{2}\left|x_{r_{1}}-x_{r_{2}}\right|^{2}\right)}\right)\right\}
\end{aligned}
$$

where $Q(u)=\frac{1}{\sqrt{2 \pi}} \int_{u}^{\infty} \exp \left(-\frac{t^{2}}{2}\right) d t$ is the Gaussian Qfunction. The expectation in (6) is with respect to the channel state information (CSI). Using the special property of the Gaussian Q-function as $Q(u)=\frac{1}{\pi} \int_{0}^{\pi / 2} \exp \left(-\frac{u^{2}}{2 \sin ^{2} \theta}\right) d \theta$ and averaging over the exponential distribution of the squared magnitude of the channel gains, it can proved that the $P S E P_{r}$ is given by

$$
\begin{aligned}
& P S E P_{r}= \\
& \frac{1}{\pi} \int_{0}^{\pi / 2} \frac{1}{\left(1+\frac{P_{s} \delta_{s, d}^{2}\left|x_{s_{1}}-x_{s_{2}}\right|^{2}}{4 N_{0} \sin ^{2} \theta}\right)} \cdot \frac{1}{\left(1+\frac{P_{r} \delta_{r, d}^{2}\left|x_{r_{1}}-x_{r_{2}}\right|^{2}}{4 N_{0} \sin ^{2} \theta}\right)} d \theta .
\end{aligned}
$$

An upper bound on $P S E P_{r}$ can be obtained by neglecting the one term in the denominator of the terms inside the integration of (7). The $P S E P_{r}$ can now be upper bounded as

$$
P S E P_{r} \leq \frac{3 N_{0}^{2}}{\delta_{s, d}^{2} \delta_{r, d}^{2} P_{s} P_{r}\left|x_{s_{1}}-x_{s_{2}}\right|^{2}\left|x_{r_{1}}-x_{r_{2}}\right|^{2}} .
$$

In order to minimize the PSEP, symbols that have adjacent constellation points in the source constellation are assigned nonadjacent constellation points in the relay constellation assignment and vice versa. So instead of using repetition at the relay node (i.e., using the same constellation as the source node) we can do constellation reassignment at the relay node to better separate the symbols to maximize the product $\left|x_{s_{1}}-x_{s_{2}}\right|^{2}\left|x_{r_{1}}-x_{r_{2}}\right|^{2}$. The constellation reassignment scheme can improve the system PSEP performance, and hence the SER performance, without increasing the complexity of the system. The proposed constellation reassignment scheme can be thought of as some form of complex field coding.

The most common constellations used in communication systems are BPSK, QPSK, 16-QAM, and 64-QAM. For BPSK, constellation reassignment at the relay nodes is meaningless since the BPSK constellation has only two points. For QPSK, the performance gains of using constellation reassignment are not significant and we do not show the results for that case.

For 16-QAM and 64-QAM constellations, one possible way to do the constellation reassignment at the relay nodes is to use exhaustive search over all the possible relay constellation assignments and select the one that maximizes the minimum value of the product $\left|x_{s_{1}}-x_{s_{2}}\right|^{2}\left|x_{r_{1}}-x_{r_{2}}\right|^{2}$ over all the possible pairs of transmitted symbols. However, the exhaustive search is extremely complex, for example, for the single-relay case, the number of possible constellation assignments at the relay node is $16 !=2.0923 \times 10^{13}$ for 16 -QAM constellation and $64 !=1.2689 \times 10^{89}$ for $64-\mathrm{QAM}$ constellation. This renders the exhaustive search impractical for constellation reassignment at the relay node. Therefore, we resort to the use of heuristic approaches for constellation reassignment at 


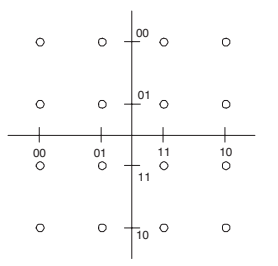

(a) Source constellation

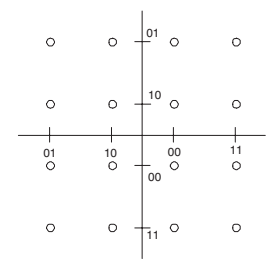

(b) Relay constellation
Fig. 2. Trans-modulation for 16-QAM constellation.

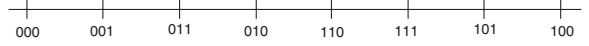

(a) Source constellation (real axis)

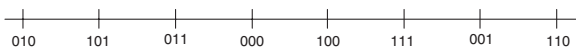

(b) Relay constellation (real axis)

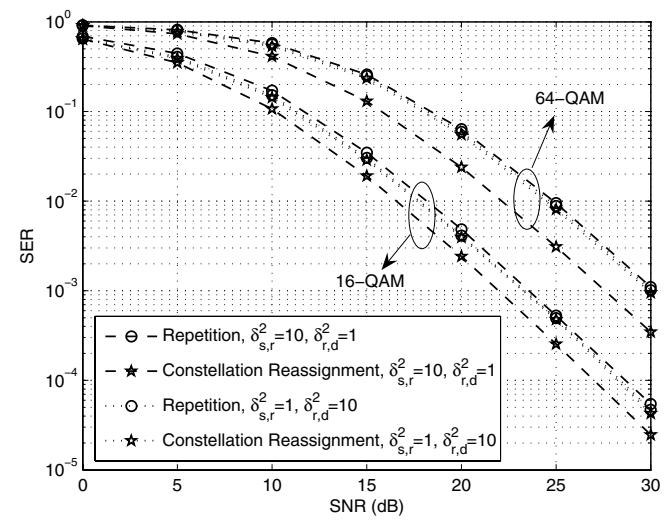

Fig. 4. SER for single-relay DAF system using 16-QAM and 64-QAM constellations.

For the case of relay close to destination we can observe that, for both 16-QAM and 64-QAM constellations, there is no significant performance gain of using the constellation reassignment scheme as compared to the repetition based scheme. In this case, and under relay transmission, the distance between two possible transmitted symbols will be dominated by the distance resulting from the relay constellation. Transmitted symbols are most likely to be mistaken with their adjacent symbols in the relay node constellation.

\section{CONCLUSIONS}

In this letter, we consider the use of constellation reassignment at the relay nodes to improve the system SER performance. The constellation reassignment is based on separating symbols with adjacent constellation points in the source constellation. This constellation reassignment increases the Euclidean distance between the closest possible transmitted symbols and hence, improves the system SER performance. Also, the proposed constellation reassignment scheme does not increase the complexity of the system. For the case of relays close to source, we can achieve an improvement of about 2 $\mathrm{dB}$ for 16-QAM constellation and about $3 \mathrm{~dB}$ for 64-QAM constellation.

\section{REFERENCES}

[1] J. N. Laneman, D. N. C. Tse, and G. W. Wornell, "Cooperative diversity in wireless networks: Efficient protocols and outage behavior," IEEE Trans. Inform. Theory, vol. 50, no. 12, pp. 3062-3080, Dec. 2004.

[2] J. N. Laneman and G. W. Wornell, "Distributed space-time coded protocols for exploiting cooperative diversity in wireless networks," IEEE Trans. Inform. Theory, vol. 49, no. 10, pp. 2415-2425, Oct. 2003.

[3] A. K. Sadek, W. Su, and K. J. R. Liu, "Multi-node cooperative communications in wireless networks," IEEE Trans. Signal Processing, vol. 55 , pp. 341-355, Jan. 2007.

[4] H. Samra, Z. Ding, and P. M. Hahn, "Optimal symbol mapping diversity for multiple packet transmission," in Proc. IEEE International Conference on Acoustics, Speech and Signal Processing (ICASSP), Apr. 2003.

[5] C. Wengerter, A. Elbwart, and E. Seidel, "Constellation rearrangement: enhancement for multilevel modulation formats and transmit diversity," Wireless Personal Communications, Kluwer Academic Publishers, vol. 29 , no. 1-2, pp. 35-45, Apr. 2004.

[6] W. Su, A. K. Sadek, and K. J. R. Liu, "Cooperative communications in wireless networks: performance analysis and optimum power allocation," Wireless Personal Commun., vol. 44, no. 2, pp. 181-217, Jan. 2008.

\footnotetext{
${ }^{1}$ The proposed constellation for 16-QAM in Fig. 2 can be proved to be optimal along one axis (real or imaginary), i.e., it is the optimal if we do the optimization (exhaustive search) along the real axis and then the imaginary axis. But, it is not guaranteed to be the global optimum if we consider optimization along both axes simultaneously.
} 